\title{
Prolonged Perceptual Learning of Positional Acuity in Adult Amblyopia: Perceptual Template Retuning Dynamics
}

\author{
Roger W Li, ${ }^{1,2}$ Stanley A Klein, ${ }^{1}$ and Dennis M Levi ${ }^{1}$ \\ ${ }^{1}$ School of Optometry and the Helen Wills Neuroscience Institute, University of California, Berkeley, Berkeley, California 94720-2020, and ${ }^{2}$ Department of \\ Psychology, The University of Hong Kong, Pokfulam, Hong Kong
}

\begin{abstract}
Amblyopia is a developmental abnormality that results in physiological alterations in the visual cortex and impairs form vision. It is often successfully treated by patching the sound eye in infants and young children, but is generally considered to be untreatable in adults. However, a number of recent studies suggest that repetitive practice of a visual task using the amblyopic eye results in improved performance in both children and adults with amblyopia. These perceptual learning studies have used relatively brief periods of practice; however, clinical studies have shown that the time-constant for successful patching is long. The time-constant for perceptual learning in amblyopia is still unknown. Here we show that the time-constant for perceptual learning depends on the degree of amblyopia. Severe amblyopia requires $>50 \mathrm{~h}(\approx 35,000$ trials $)$ to reach plateau, yielding as much as a five-fold improvement in performance at a rate of $\approx 1.5 \% / \mathrm{h}$. There is significant transfer of learning from the amblyopic to the dominant eye, suggesting that the learning reflects alterations in higher decision stages of processing. Using a reverse correlation technique, we document, for the first time, a dynamic retuning of the amblyopic perceptual decision template and a substantial reduction in internal spatial distortion. These results show that the mature amblyopic brain is surprisingly malleable, and point to more intensive treatment methods for amblyopia.
\end{abstract}

Key words: plasticity; critical period; visual learning; positional acuity; classification image; amblyopia

\section{Introduction}

Amblyopia (from the Greek, amblyos: blunt; opia: vision) is a developmental disorder that results in physiological alterations in the visual cortex and impairs form vision (Levi et al., 1991), and is usually associated with abnormal visual experience caused by strabismus, anisometropia and visual deprivation early in life. Aside from refractive error, amblyopia is one of the main causes for the loss of vision in children (Newman and East, 2000). Clinically, it is often successfully treated by patching the sound eye in infants and young children (occlusion therapy), but is generally considered to be untreatable in adults and older children who fall outside the period of cortical plasticity. A long period of occlusion, ranging from several months to a year, is needed for the recovery of amblyopic vision, with acuity improving $\sim 0.22 \%$ per hour of occlusion (Stewart et al., 2004), and the dose-response appears to plateau only after 100-400 h (Cleary, 2000; Stewart et al., 2004, 2005).

Although treatment of amblyopia is generally not applied to adults, it is now clear that adults with amblyopia can improve following perceptual learning, i.e., repetitive practice of a demanding visual task (Levi and Li, 2008). Over a decade ago, Levi and Polat first showed that practice enhanced Vernier acuity in

Received Sept. 7, 2008; accepted Nov. 8, 2008.

This work was supported by National Eye Institute Grants R01EY01728 (D.M.L.) and R01EY04776 (S.A.K.) and a James S. McDonnell Foundation grant (D.M.L.).

Correspondence should be addressed to Roger Li, School of Optometry, University of California, Berkeley, Berkeley, CA 94720-2020. E-mail: oroger@berkeley.edu.

DOI:10.1523/JNEUROSCI.4271-08.2008

Copyright $\odot 2008$ Society for Neuroscience $\quad$ 0270-6474/08/2814223-07\$15.00/0 adults with amblyopia and that this improvement transferred to improvement in visual acuity in some of their observers (Levi and Polat, 1996; Levi et al., 1997). It is now clear that perceptual learning improves amblyopic performance on a wide range of tasks, including position discrimination (Li and Levi, 2004), spatial interaction (Polat et al., 2004), contrast detection (Zhou et al., 2006), and letter recognition (Levi, 2005; Chung et al., 2006, 2008).

Most previous learning studies used relatively brief periods of practice (Li and Levi, 2004; Li et al., 2005) as it is generally believed that the adult brain has limited plasticity and thus perceptual learning in normal foveal vision is rapidly completed after a few hours of practice. For example, Levi and Polat (Levi and Polat, 1996; Levi et al., 1997) had observers complete only $4000-$ 5000 practice of trials $(\approx 5-10 \mathrm{~h}$ ). Li and Levi asked seven amblyopic patients to practice a positional discrimination task and they seemed to have reached the plateau level and showed a modest improvement $(20-30 \%)$ in a brief period $(\approx 20 \mathrm{~h})$ of training $(\mathrm{Li}$ and Levi, 2004; Li et al., 2005). Other studies used a criterion of asymptotic performance on three consecutive sessions, giving a mean length of training of $\approx 12$ sessions, or $12 \mathrm{~h}$ (Zhou et al., 2006; Huang et al., 2008). The most extensive training was provided by Polat et al. Their subjects practiced a contrast detection task for, on average 45 sessions, $\approx 22 \mathrm{~h}$ (Polat et al., 2004). Interestingly, close inspection of their data, as well as that of the other studies, suggests that observers may still have not reached their ultimate asymptotic performance. This raises the question of whether "prolonged" perceptual learning would result in more substantial improvement in adults with amblyopia.

A recent study revealed that in two juvenile amblyopes, pro- 
Table 1. Clinical data

\begin{tabular}{|c|c|c|c|c|c|c|c|c|}
\hline Observer & Age (years) & Gender & Cover test & Eye & Refractive error & Snellen VA & Stereopsis (arcsec) & Type of amblyopia \\
\hline SF & 18 & $M$ & $6^{\Delta}$ LExoT & $\begin{array}{l}\mathrm{R} \\
\mathrm{L}\end{array}$ & $\begin{array}{l}-1.50 /-0.25 \times 90 \\
\text { Plano/-1.00 × } 30\end{array}$ & $\begin{array}{l}20 / 12.5^{+1} \\
20 / 125^{-2}\left(20 / 80^{+2}\right)\end{array}$ & Failed $(>400)$ & Strab \\
\hline JS & 22 & $\mathrm{~F}$ & $\begin{array}{l}7^{\Delta} \text { LEsoT } \\
5^{\Delta} \text { L HyperT }\end{array}$ & $\begin{array}{l}\mathrm{R} \\
\mathrm{L}\end{array}$ & $\begin{array}{l}+1.00 \\
+0.75\end{array}$ & $\begin{array}{l}20 / 16 \\
20 / 32^{+1}\left(20 / 20^{-1}\right)\end{array}$ & 70 & Strab \\
\hline $\mathrm{AA}$ & 29 & $\mathrm{~F}$ & $\begin{array}{l}>30^{\Delta} \text { Alt EsoT } \\
10^{\Delta} \text { R HyperT }\end{array}$ & $\begin{array}{l}\mathrm{R} \\
\mathrm{L}\end{array}$ & $\begin{array}{l}-2.00 /-2.25 \times 180 \\
-3.75 /-2.00 \times 5\end{array}$ & $\begin{array}{l}20 / 25^{-2}\left(20 / 20^{+2}\right) \\
20 / 20^{+2}\end{array}$ & Failed $(>400)$ & Strab \\
\hline SC & 29 & M & NMD & $\begin{array}{l}\mathrm{R} \\
\mathrm{L}\end{array}$ & $\begin{array}{l}+0.50 \\
+3.25 /-0.50 \times 155\end{array}$ & $\begin{array}{l}20 / 16^{+2} \\
20 / 50^{+2}\left(20 / 40^{-2}\right)\end{array}$ & Failed $(>400)$ & Aniso \\
\hline ED & 39 & M & NMD & $\begin{array}{l}\mathrm{R} \\
\mathrm{L}\end{array}$ & $\begin{array}{l}+2.25 /-1.00 \times 70 \\
+0.25 /-0.25 \times 110\end{array}$ & $\begin{array}{l}20 / 32\left(20 / 32^{+1}\right) \\
20 / 16^{+2}\end{array}$ & 25 & Aniso \\
\hline VC & 22 & $\mathrm{~F}$ & $5^{\Delta}$ RExoT & $\begin{array}{l}\mathrm{R} \\
\mathrm{L}\end{array}$ & $\begin{array}{l}+4.75 /-1.50 \times 10 \\
\text { Plano/- } 0.50 \times 170\end{array}$ & $\begin{array}{l}20 / 125^{-4}\left(20 / 125^{-1}\right) \\
20 / 12.5^{-2}\end{array}$ & Failed $(>400)$ & Strab and Aniso \\
\hline AW & 23 & $\mathrm{~F}$ & $\begin{array}{l}5^{\Delta} \text { R ExoT } \\
4^{\Delta} \text { L HyperT }\end{array}$ & $\begin{array}{l}\mathrm{R} \\
\mathrm{L}\end{array}$ & $\begin{array}{l}+2.75 /-1.00 \times 160 \\
-1.00 /-0.50 \times 180\end{array}$ & $\begin{array}{l}20 / 80^{-1}\left(20 / 50^{-1}\right) \\
20 / 16^{-1}\end{array}$ & Failed $(>400)$ & Strab and Aniso \\
\hline BK & 24 & M & NMD & $\begin{array}{l}\mathrm{R} \\
\mathrm{L}\end{array}$ & $\begin{array}{l}-6.25 /-1.25 \times 10 \\
-7.00 /-1.25 \times 160\end{array}$ & $\begin{array}{l}20 / 20^{+2} \\
20 / 20^{+2}\end{array}$ & 20 & Normal \\
\hline DW & 22 & $\mathrm{~F}$ & NMD & $\begin{array}{l}\mathrm{R} \\
\mathrm{L}\end{array}$ & $\begin{array}{l}\text { Plano/- } 0.50 \times 10 \\
\text { Plano }\end{array}$ & $\begin{array}{l}20 / 16^{-1} \\
20 / 16^{-2}\end{array}$ & 20 & Normal \\
\hline
\end{tabular}

Seven adults with strabismic (Strab) and/or anisometropic (Aniso) amblyopia participated. Their visual acuity (VA) in the amblyopic eye ranged from $\approx 20 / 25$ (mild) to $\approx 20 / 125$ (severe), and their isolated letter acuity is listed in parenthesis. M, Male; F, female; R, right; L, left; ExoT, exotropia; EsoT, esotropia; Hyper T, hypertropia; Alt, alternate; NMD, no movement detected.

longed perceptual learning yielded an $\sim 2.4$-fold improvement in performance after $50 \mathrm{~h}$ of training ( $\mathrm{Li}$ et al., 2007). One might argue that the developing brain is more malleable, thus a longer period of training can produce more substantial improvements. But it raises the important question of whether or not similar prolonged perceptual learning would result in more substantial gains in adult amblyopes. We know that practicing position discrimination can reduce spatial distortion (internal positional noise) and enhance the ability to extract stimulus information (sampling efficiency) during the course of training (Li and Levi, 2004; Li et al., 2005, 2007). However, there remains a longstanding fundamental question of how the amblyopic brain retunes its abnormal neuronal connections so as to optimize and sharpen its responses to the visual world.

To address these questions, we adopted a much stricter stopping rule than in previous studies to decide whether training should continue, and our experiment was designed to explore the amount of experience-dependent plasticity in the mature amblyopic brain. Position discrimination depends strongly on spatial relations, and is thought to be limited by positional uncertainty in amblyopes. Thus, in this study we used "positional" noise, i.e., perturbation of the positions of parts of the stimulus, to mimic the putative limiting internal noise by rendering the positions of the samples uncertain (rather than by obscuring their visibility, as occurs with luminance noise) and to explore the underlying neural mechanisms for position discrimination. Our results reveal surprisingly substantial cortical plasticity in adult amblyopia following a prolonged period of repetitive visual stimulation. Using a reverse correlation technique, we were able to document, for the first time, the session-to-session retuning dynamics of the perceptual decision template or perceptive fields of the amblyopic brain during visual learning. An understanding of the limits, time course, and mechanisms of perceptual learning is critical for developing a more effective amblyopia treatment.

\section{Materials and Methods}

Experimental procedures. Seven adults aged 18-40 years with anisometropic and/or strabismic amblyopia participated in the study (Table 1). They practiced the positional discrimination task repeatedly using their amblyopic eye; the other eye was occluded with a standard black eye patch. Each day's session consisted of 960 trials in $\sim 1.5$ h, $3-5$ sessions a week.

To evaluate the possible "maximum" limits of cortical plasticity, we adopted a much stricter criterion for ending the training course than those in our previous study (Li and Levi, 2004); our observers were required to continue practicing the visual task until their improved performance were stable and maintained for as much as 15-20 sessions after the plateau levels were reached. Consequently, three of our observers had given $>50,000$ responses in 50 training sessions. All observers were naive to the purpose of experiment.

Visual stimulus. The stimulus was comprised of two horizontal segments with a $1.25 \lambda$ gap between the centers of the innermost patches (see Fig. 1A). Each segment consisted of five Gabor patches, and the interpatch separation was $0.83 \lambda$. The patches were constructed to have a $2 / 3$ aspect ratio: the Gaussian envelope SD was 0.39 and $0.58 \lambda$ for the horizontal and vertical orientations respectively. Positional noise was produced by shifting the position of each Gabor patch in the vertical direction around the intended mean line position of the test (right) segment according to a discrete binary probability function. The binary noise amplitude was always $0.11 \lambda$, either positive (downward) or negative (upward). No noise was added to the reference (left) segment. The carrier spatial frequency, and stimulus size, was scaled [5 (VC and AW), 6.6 (SF), or 10 (SC, JS, ED, AA, and normal observers) cycles per degree] according to observer's baseline visual acuity of the amblyopic eye by varying viewing distance $(4,2$, or $1 \mathrm{~m})$, without changing any physical dimensions of the stimulus displayed on the monitor screen.

The stimulus was briefly presented $(200 \mathrm{~ms})$ on a flat 21 -inch Sony F520 monitor screen at $90 \mathrm{~Hz}$ refresh rate. Subjects were asked to maintain fixation at the center of the monitor screen. The mean luminance of the stimuli was $55 \mathrm{~cd} / \mathrm{m}^{2}$, and the contrast of each Gabor patch was $84 \%$.

Threshold calculation. On each trial, the test line was presented randomly in one of three positions: aligned with the reference line, or one step above $(-)$ or below $(+)$ it. The observers' task was to rate the position of the test segment compared with the reference segment by giving an integer number from -2 (above) to 2 (below), including 0 (aligned). Trial-by-trial verbal feedback was provided following each trial. Observers were instructed to attend to all five Gabors in each segment so as to determine the average segment positions and detect the direction of misalignment. A rating-scale signal detection paradigm was used to calculate $d^{\prime}$ (detectability or effect size) for discriminating the direction of offset (Levi et al., 2000). The position offset at which $d^{\prime}=1$ was taken as threshold. At the start of each training session, the offset between the two segments was adjusted according to the previous ses- 
A

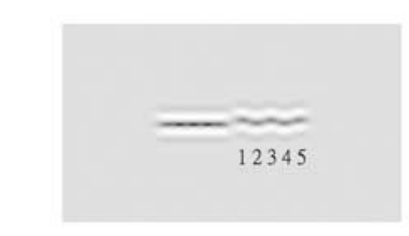

G
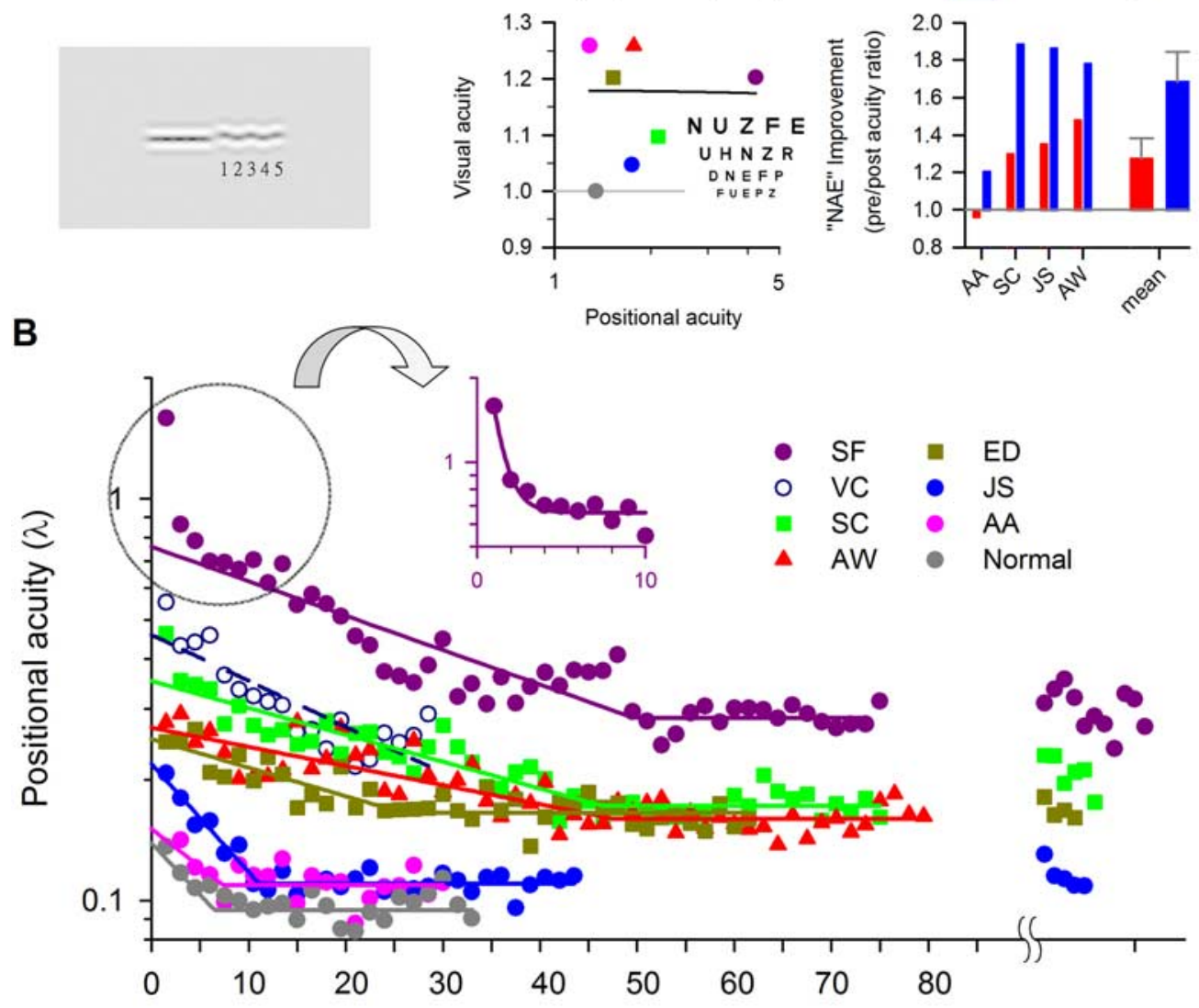

Hours of practice
$\mathbf{F}$

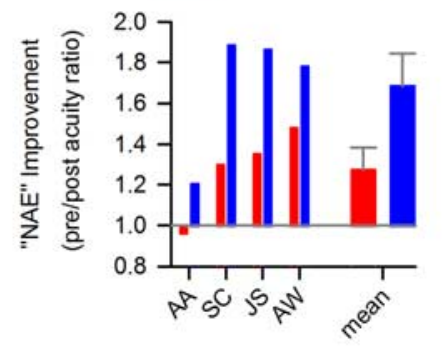

D

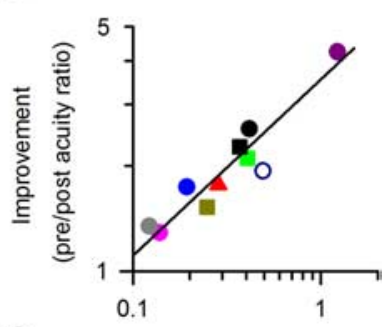

C

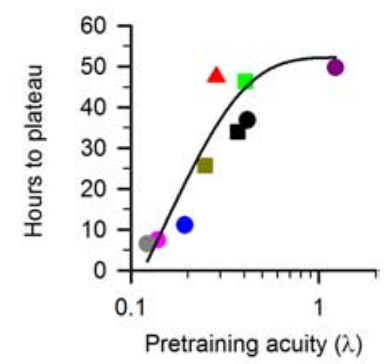

Figure 1. Prolonged perceptual learning of position discrimination in adult amblyopia. $A$, Visual stimulus. The observers' task was to identify the location of the right test segment relative to the left reference segment. Positional noise was added to the right segment by jittering the vertical position of each of the five Gabor patches (either up or down). $\boldsymbol{B}$, Learning profile. Each data point represents one session consisting of 960 trials in $\sim 1.5 \mathrm{~h}$. It is worth noting that it took as many as $\approx 35$ sessions $(\approx 35,000$ trials) to improve and reach a stable performance plateau (prolonged learning). Observer V ( withdrew from the study after 19 sessions (open symbols in this and subsequent figures) and did not finish the experiment according to our end-point criterion. Gray symbols indicate the mean data of two normal observers for comparison. C, The number of hours needed to reach stable plateau performance as a function of pretraining positional threshold. D, Acuity improvement (pre/post ratio) as a function of pretraining positional threshold. The data of two children (black symbols in panels $\boldsymbol{C}$ and $\boldsymbol{D}$ ) with amblyopia who underwent intensive perceptual learning are replotted here (Li et al., 2007). $E$, Comparison of positional acuity between the two eyes, nonamblyopic (NAE) and amblyopic (AE). The posttraining performance (filled symbols) is much closer to (or even better than) the gray 1:1 reference line. The amount of plateau improvement, as indicated by the length of colored lines, is considerably larger for those observers with much elevated baseline pretraining threshold (open symbols). $\boldsymbol{F}$, Interocular transfer (from the trained AE to the nontrained fellow NAE) versus phase II direct training (on the previously untrained NAE). $G$, Improvement in visual acuity and positional acuity. The amount of improvement in visual acuity is not dependent on that in positional acuity (slope $=-0.0015 \pm 0.04, t=-0.04, p=0.97$ ).

sions' thresholds so as to keep the detectability around 1, aiming to make the task challenging, not too easy or too difficult.

The decision template and internal noise. The details of decision template (also known as classification image) and internal noise calculations are fully described elsewhere ( $\mathrm{Li}$ et al., 2006). This template provides the decision rules of how the brain extracts and integrates stimulus information for positional judgments. In brief, a multiple linear regression was used to reverse correlate the individual positions of the 5 Gabors (see Fig. $1 A$ ) with the human responses $(-2$ to +2$)$ in the computation of perceptive fields, with the sum of the five normalized weightings equal to unity. For each of three offset levels $(-, 0$, and +$)$, there are $2^{5}=32$ possible external noise combinations in total.

Each stimulus arrangement was presented 10 times to observers, therefore each session consisted of 960 trials ( 32 noise combinations $\times 3$ offset levels $\times 10$ passes) in a random sequence. The 10-pass response consistency for each noise combination provides a measure of internal noise. Importantly, the use of binary noise allows us to monitor fewer moving parts, thus requiring much fewer trials when combined with multiple regression to compute perceptive fields.

Transfer experiment. At the commencement of the training experiment, we also tested the baseline performance of the fellow nonambly- opic eye at the same viewing distance as selected for testing the amblyopic eye. After Phase 1 training on the amblyopic eye, we switched to train the nonamblyopic eye in Phase 2 training to evaluate whether there was any interocular transfer (complete or incomplete) from the trained amblyopic eye to the untrained fellow eye.

Clinical vision tests. In this study, a Bailey-Lovie logMAR letter chart (National Vision Research Institute of Australia, 1978) was used to measure visual acuity, and Randot Stereotest (Stereo Optical Company) was used to measure stereoacuity for the range of $20-400 \mathrm{arcsec}$.

\section{Results}

\section{Prolonged perceptual learning in adult amblyopia}

Most previous studies (Levi and Polat, 1996; Li and Levi, 2004; Dosher and $\mathrm{Lu}, 2007$ ) have used either a fixed duration of practice (typically $10-20 \mathrm{~h}$ or $4000-8000$ trials) or a stopping criterion of asymptotic performance on three consecutive sessions (Zhou et al., 2006). However, our results suggest the need for a much stricter stopping rule. Extended perceptual learning results in a more substantial, although not complete recovery in positional threshold (Fig. $1 B$ ). Practice improves positional acuity 
(the minimum offset between the two segments to be detected decreases across training sessions) gradually until plateau performance is obtained. A three parameter two-line segment curve was used to fit the logarithmic threshold data as a function of training session; the intersection point of the two lines indicates the plateau performance for each observer. Note that the threshold data are plotted in Gabor wavelength $(\lambda)$ units to facilitate the comparison between the different viewing distances used to test individual observers. Within this extended period of improvement there may be short plateaus in performance (Fig. $1 B$, inset), followed by further improvement.

Interestingly, observers with mild amblyopia improve at a faster rate than those with severe amblyopia, as reflected by a steeper regression slopes in Figure $1 B$ [mean improvement rate: mild (JS and AA) $11.4 \pm 1.9 \% / \mathrm{h}$; severe (SF, VC, SC, and AW) $4.0 \pm 0.7 \% / \mathrm{h}]$. More practice is needed to reach asymptote in severe amblyopia than in mild amblyopia (Fig. 1C), and the amount of improvement is directly proportional to the severity of amblyopia on a log-log scale (Fig. $1 D)$. The pre/post acuity ratio was larger than a factor of 4 in a severe amblyope (SF). In general, deep amblyopes [SF, SC and AW (visual acuity, VA): 20/50-20/ $125)]$ required $\approx 50 \mathrm{~h}(\approx 35,000$ trials $)$ to reach plateau, and the acuity ratio was as much as $1.75-4.23$-fold. In contrast, mild amblyopes [ED, JS and AS (VA: 20/25-20/40)] required fewer practice trials $(7.5-30 \mathrm{~h})$ to obtain stable improvement (1.351.75 -fold). The exponential time-constants were 18.75 and $5.55 \mathrm{~h}$ for deep and mild amblyopes respectively and just $2.7 \mathrm{~h}$ for normal control observers. The black symbols in Figures 1, $C$ and $D$, show data of two children (ages 8 and 11) with amblyopia who underwent intensive perceptual learning. While the stimulus configurations and psychophysical methods were different, these data provide a close match with the adult data, suggesting that age (at least from 8 to 39) is not an important determinant of the outcome of perceptual learning.

The posttraining threshold in the amblyopic eye (solid symbols) approaches, and even crosses the 1:1 line representing equality of threshold in the amblyopic and nonamblyopic eyes (Fig. 1E). Note that one observer (VC, dashed line) did not complete the training according to our stopping criterion; thus it is possible that additional practice could result in further improvement. Interestingly in three mild amblyopes (AA, JS, and ED), plateau performance of the trained amblyopic eye was even better than the baseline performance of the untrained "normal" eye. In other words, the amblyopic deficits for this type of positional acuity are more or less eliminated.

An important question is whether the improved performance is maintained after cessation of training. We retested four observers on the same task after a period of 1-2 months and found that all or most of the improved performance was retained (the rightmost symbols of Fig. $1 \mathrm{~B}$ ). Although there was a small elevation in threshold $(6.5 \% \pm 1.8 \%$ when compared with the baseline measurement), only a few $(\approx 5)$ maintenance sessions were enough to achieve the original plateau levels. Note that after direct training on the amblyopic eye, two of those four observers (SC and JS) elected to proceed in training the nonamblyopic eye for a month (interocular transfer experiment) immediately before retesting the amblyopic eye. One might speculate that the learning effects, if any, in the nonamblyopic eye could possibly transfer, to a certain extent, back to the amblyopic eye. Therefore, we retested the maintenance performance in another two observers (SF and ED) who did not participate in the transfer experiment 1-2 months after the last session of visual testing and confirmed that the improved performance was long-lasting and substantially maintained.

\section{The roles of higher- and lower-level processing in learning}

One way to reveal where neural alternations occur is to evaluate interocular transfer of learning. An absence of transfer from the trained eye to the untrained eye is often taken as evidence for neural alternations occurring at a stage of visual processing before binocular convergence. Alternatively, a complete transfer would be expected if higher cortical areas are involved. Our findings (Fig. $1 F$ ) show a significant transfer of learning to the sound eye in 3 of the four observers (mean pre/post acuity ratio = $1.27 \pm 0.11, t=2.458, p=0.046)$. Interestingly, with further practice the nonamblyopic eye showed additional improvement, mean pre/post acuity ratio $=1.68 \pm 0.16$ (mean difference in acuity ratio $=0.41 \pm 0.08$, paired $t=4.995, p=0.015)$. This suggests that training the amblyopic eye modified the response characteristics of higher visual cortex which in turn results in the interocular transfer, and the subsequent training of the fellow eye itself may have further strengthened early neural mechanisms. Recent physiological studies also reported the involvement of higher decision stage in visual learning (Law and Gold, 2008). These effects may also explain the accompanying generalized transfer to improved visual (letter) acuity (Fig. $1 G$ ), the sine qua non of amblyopia, consistent with the recent finding that perceptual learning has a broad bandwidth in amblyopia (Huang et al., 2008)

\section{Retuning dynamics of behavioral receptive fields}

Using an efficient trial-by-trial reverse correlation method ( $\mathrm{Li}$ et al., 2004, 2006), we are able to monitor changes, if any, in the decision template or "behavioral" receptive field (Gold et al., 1999) for positional judgments during visual learning. The decision template reveals how observers weight and integrate the positional information of the 5 patches comprising the test segment of the visual stimulus (Fig. 1A). An "ideal" observer who "knows" the stimulus exactly would give equal weight $(0.2)$ to each patch for positional averaging (Fig. $2 \mathrm{~A}$, inset). Not surprisingly, human observers adopt a less efficient template for extracting positional information; the averaging computation relies on a limited number of stimulus samples.

Importantly, initially the amblyopic eye shows distinct differences in positional integration from the fellow sound eye (Fig. $2 B$ ): (1) The peak of tuning is shifted to the right (away from the inner segment) in observers SC (patch 4), SF (patch 5) and VC (patch 5). In fact, SF's and VC's responses are mostly based on the position of the outermost patch. This may reflect abnormal suppression, or crowding (Nandy and Tjan, 2007; Levi, 2008) effects of the reference (left) segment on the proximal samples of the test (right) segment. In contrast, their nonamblyopic eyes used a more efficient ideal-observer-like template. (2) With our abutting stimulus, one might expect that amblyopic observers would down-weight the first (inner) patch because of crowding effects. In contrast, we observed a very strong response, but in the wrong direction: repulsion error [patch 1: SC $(-0.37)$, SF $(-1.11)$, and VC (-0.83); ideal observer (0.2)], representing a misperception of position (or spatial distortion): when the patch is up, the perceived position is down, and vice versa. It is widely known that spatial interaction affects contrast (Polat et al., 2005) and contour (Hess et al., 2001) perception, and here is another example of abnormal interaction for positional perception. (3) In an extreme case, severe amblyopes SF and VC adopted an ideal-observer like template [note that the gray open (VC, $80.6 \%$ efficiency as illus- 


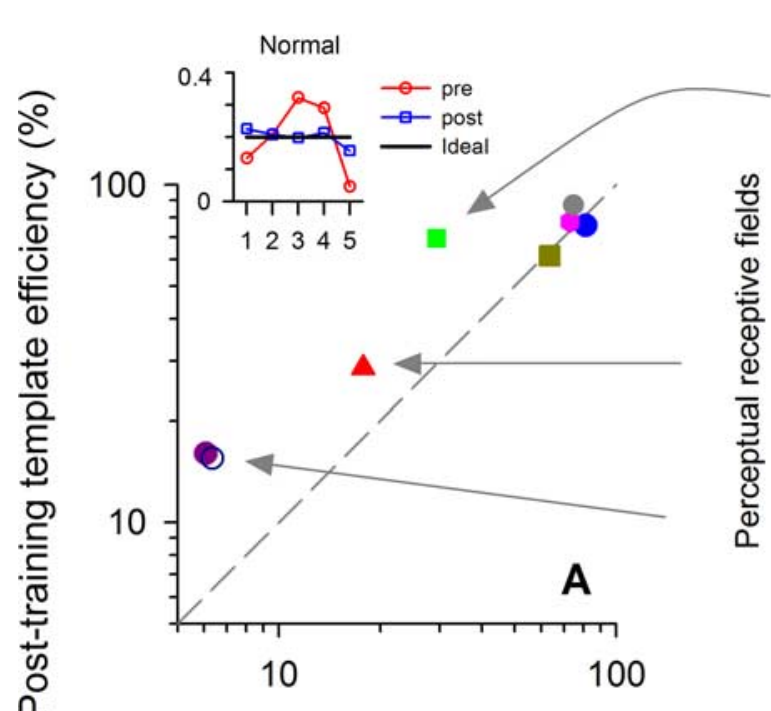

Pre-training template efficiency (\%)

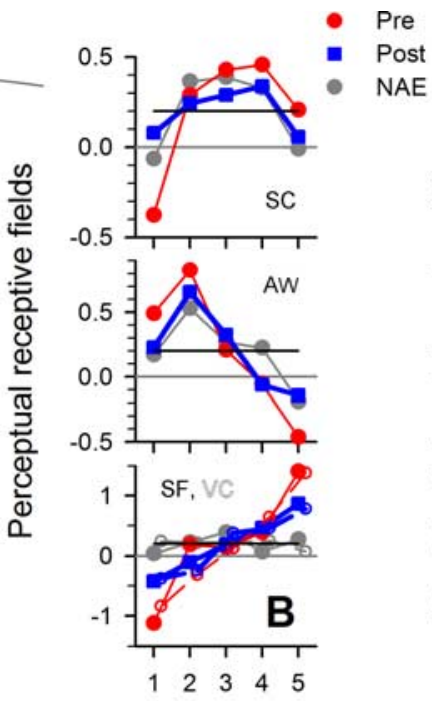

Patch position

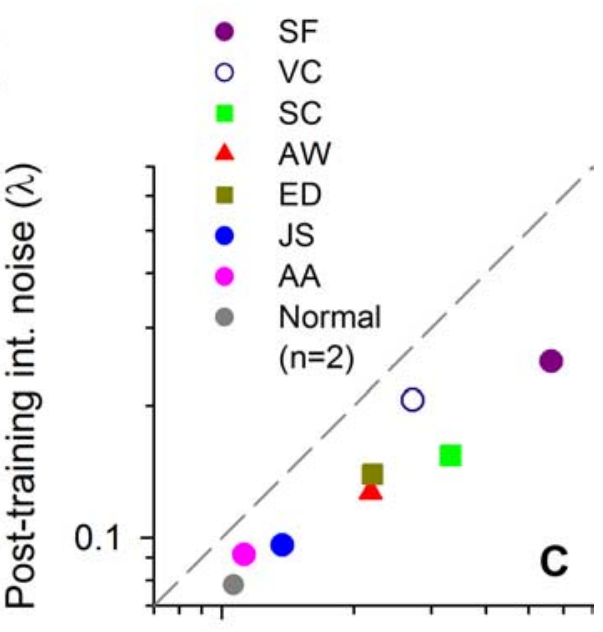

0.1

Pre-training int. noise $(\lambda)$

Figure 2. Neural mechanisms involved in learning position discrimination. $\boldsymbol{A}$, Inset, Ideal versus human template. In human observers ( $n=2$ ), the averaging computation mostly relied on a few stimulus samples; much higher weights were given to the selected samples than to the others (red line). Following practice, the retuned template (blue line) is closer to resembling the ideal template (black line). $\boldsymbol{A}$, The enhancement of template efficiency after perceptual learning. In four deeply amblyopic observers, the posttraining efficiency is much higher than the pretraining efficiency. Nevertheless, the other three mild amblyopes (ED, JS, and AA) did not show any significant retuning, as shown by similar pretraining and posttraining efficiency along a $1: 1$ reference line. $\boldsymbol{B}$, Template retuning. A red line illustrates the pretraining template and a blue line illustrates the posttraining template instead. The retuned template is more similar to the ideal template in shape (black line). For comparison, the data of the nonamblyopic eye (NAE) is plotted as a gray line. C, Perceptual learning results in a decrease in random internal (int.) noise. It appears that the amount of decrement is directly proportional to the baseline noise level. Note that observer VC (open symbols) did not complete the experiment according to our plateau criterion.

trated in Fig. 2A) and solid (SF, 67.4\% efficiency) symbols fall close to the black horizontal line for ideal perceptual fields] in the fellow sound eyes, but the least efficient template of only $\sim 6 \%$ efficiency (a very tilted oblique template profile placing unequal weights for different parts of stimulus with an abnormal very large repulsion error at one end) in the amblyopic eyes. (4) However, two other amblyopes with mild loss in visual acuity (JS and AA) showed nearly normal templates (efficiency: 70-80\%) which are very comparable with the two normal observers (efficiency: 75\%).

Most importantly, we recorded a dramatic optimization of the decision template which, in part, explains the enhanced performance following practice, making the posttraining template (Fig. $2 B$ ) more similar in shape to both the nonamblyopic eye and the ideal template. On average, the template efficiency (squared correlation between human and ideal templates) improved substantially by $126.3 \%$ for those four deep amblyopes (Fig. $2 A$ ). The retuned template is capable of more effective sampling with much reduced repulsion [e.g., SF: $-1.12 \rightarrow-0.42$ (patch 1)], reflecting how the brain recalibrates the weightings of neuronal connections with response feedback to use the spatial information from lower-level visual mechanisms more effectively and appropriately. In contrast, three mild amblyopes did not show any enhancement in template efficiency because they were already highly efficient (mean: $72.5 \%$, quite similar to normal controls $74.9 \%$ ), leaving little room for improvement.

Visual learning also results in a substantial reduction in internal positional noise (random jitter). We assayed internal noise by repeating each combination of signal and noise 10 times in each session. Since the signals and noise are identical, any inconsistency in responses provides an accurate assay of internal noise (Green, 1964). All observers showed a reduction of internal noise (mean: $40.2 \%$ ) in the posttraining session (expressed in $\lambda$ unit in Fig. $2 C$ to facilitate the comparison between different viewing distances used for individual observers), ranging from $18.4 \%$ (AA) to $55.2 \%$ (SF). The more severe the amblyopia, the larger the reduction (farther away from the 1:1 reference line).

We can model the observers' performance, human threshold $\sigma_{\text {th }}$ (Fig. 3B, black line), by summing the various sources of noise $\left(\sigma_{\text {th }}^{2}=\sigma_{\text {temp }}^{2}+\sigma_{\text {random }}^{2}+\sigma_{\text {systemic }}^{2}\right)$ : template noise $\left(\sigma_{\text {temp }}\right)$ due to a nonideal decision template and random $\left(\sigma_{\text {random }}\right)$ and systematic $\left(\sigma_{\text {systematic }}\right)$ internal noise beyond the template ( $\mathrm{Li}$ et al., 2006). The data are replotted in Figure $3 A$ to summarize the changes in template efficiency and random internal noise. Note that random internal noise is specified as pre/post ratio instead of post/pre ratio for template efficiency so that a ratio of $>1$ represents increased template efficiency or decreased internal noise. Figure $3 B$, which shows the "squared" threshold of each model component, reveals the dynamics of template retuning and internal noise reduction across sessions for two observers. The threshold of the ideal template, $\sigma_{\text {ideal }}$, is $0.298 \mathrm{arcmin}$ (gray area: $0.148 \lambda$ at $4 \mathrm{~m}$ viewing distance) reflecting averaging error because of stimulus positional uncertainty; the human template efficiency is defined as $E f f_{\text {Temp }}=\left(\sigma_{\text {ideal }} / \sigma_{\text {temp }}\right)^{2} \times 100 \%$. It is especially interesting to see the very gradual template retuning in observer SC (yellow area: template threshold gradually decreased until 40-45 $\mathrm{h}$ of practice; template efficiency improved substantially from $30 \%$ to $70 \%$ ), resulting in a very efficient template, only slightly less efficient than the ideal template after 35-thousand trials. In contrast, the improvement in observer ED can be mainly attributed to the reduced internal random noise (pre/post ratio: 1.58), the yellow area remains flat (pre/post ratio: 0.97 ) across the training course. The right end of each panel shows the maintenance data; it is clear that all threshold components remain much the same.

\section{Discussion}

After a prolonged period of perceptual learning positional acuity in adult amblyopia can be substantially normalized, with as much 
A

B

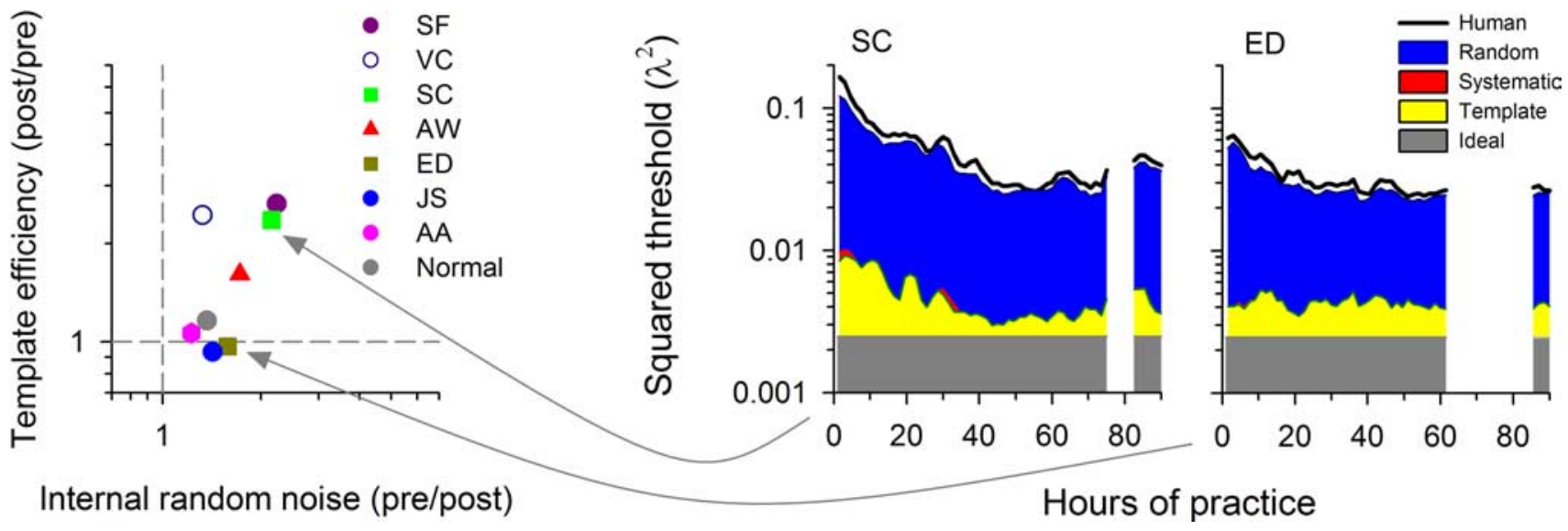

Figure 3. Retuning dynamics of behavioral receptive fields. $A$, Summary of changes in template efficiency and internal random noise. Template efficiency is expressed as post/pre ratio, while internal noise is expressed as pre/post ratio. $\boldsymbol{B}$, Threshold components. The squared sum of all threshold components (ideal template, human template, random noise, and systematic noise) is quite close to the squared human threshold, although our modeling tends to slightly overestimate human performance, as indicated by a black line. In agreement with our earlier study with normal observers (Li et al., 2006), systematic noise (like the systematic template error, but related to a higher-order nonlinearity) is negligible in this positional task.

as a five-fold improvement in performance in observers with deep amblyopia. A much longer time than expected is necessary to reach a real plateau level $(>50 \mathrm{~h})$. In normal adults, in agreement with our earlier study (Li et al., 2004), visual learning is much faster, mostly completed in just a few sessions totaling $\sim 5-10 \mathrm{~h}$ (Fig. $1 B$, gray symbols). Our findings clearly show that the mature amblyopic brain, which is generally thought to have limited capacity for neural alternations, shows substantial plasticity with prolonged perceptual learning. To our knowledge, such prolonged learning resulting in such substantial gains in visual performance, have not been previously reported in adults with normal or amblyopic vision. Importantly, the learning effects are long-lasting (Fig. $1 B$ ), and only a few retraining sessions are needed to compensate for a slight loss of previously gained improvement.

One might argue that the improvement in performance might be in part attributed to high level cognitive task learning or instrument learning (Westheimer, 2001). For example, amblyopes might learn to fixate and/or accommodate more accurately with their amblyopic eye. We argue that improvements in fixation and/or focusing accuracy are unlikely to account for our present findings for several reasons: (1) Our task (long and very visible horizontal pair of Gabor patch groupings with low/moderate carrier spatial frequency) is not strongly dependent on precise fixation or focus. (2) The very gradual, but substantial, improvement in thresholds across an extended period of training sessions indicates that the changes in sensitivity are genuine. (3) If the improvement in performance were due to improved fixation/focus, we would expect the improvement to transfer across orientations. However our previous studies using a similar positional task found no transfer across orthogonal orientations ( $\mathrm{Li}$ and Levi, 2004).

A stringent stopping rule is important for maximizing the treatment effects. As an example, when only the first 10 -sessions of SF's data are fitted with an exponential function (Fig. $1 B$, inset), it appears that the plateau performance (improvement: $51 \%$ ) would have been obtained at around 7-8 h. But further practice resulted in an additional 52\% improvement over 40 more hours. In contrast to cognitive task learning (Westheimer, 2001) that is rapid, this gradual improvement reflects genuine experience-dependent neural alternations. Similar prolonged learning effects have also been recently reported in animals with degraded auditory systems (Zhou and Merzenich, 2007).
An earlier study failed to report any correlation between the depth of amblyopia and the amount of improvement (Levi et al., 1997 ), most likely due to incomplete learning after just $\approx 10$ practice sessions. Those previous studies (Levi and Polat, 1996; Levi et al., 1997; Li and Levi, 2004) might have largely under-estimated the limits of neural plasticity in the mature amblyopic brain.

Importantly, we quantified the changes in the observers' perceptual decision templates for positional acuity as their position acuity improved. This perceptual decision template represents a spatial map that reveals how the lower-end spatial filters are calibrated, weighted and integrated for position localization. In some sense, the abnormal template before learning reflects the nature of spatial distortion and visual crowding in amblyopic vision. Here we provide evidence that the perceptual decision template of the amblyopic visual system can be retuned through repetitive practice. Thus, neuronal connections in the amblyopic brain are not completely hardwired, but are retunable. The retuned template is less distorted and more effective in interpreting positional information. Unlike the traditional averaging methods for calculating perceptive fields that require several thousands of responses, our efficient reverse correlation technique (Li et al., 2006) allows us to obtain a reliable template from only one thousand trials and to document the session-bysession retuning dynamics of the perceptual decision template during perceptual learning.

Neuronal connections of the visual system are generally thought to be not malleable into adulthood, and the question of reorganization in adult cortex following a retinal scotoma remains controversial (Smirnakis et al., 2005). However, a recent calcium imaging study has shown that rewiring can indeed occur in adult cortex following stroke (Winship and Murphy, 2008), supporting the notion that somatosensory cortical neurons still retain a remarkable degree of adaptive plasticity. Our results show that the improvement following practice with the amblyopic eye transferred to the sound eye. This result is consistent with some learning taking place at a higher "decision stage" of visual processing. It is worth noting that binocular transfer may occur as early as V1 layers 2-3 (Zhang et al., 2005); however a "more central" explanation is that at a high level decision stage, the visual system may learn to rely on (or attend to) a subset of neurons, that provide more salient signals, without necessitating physiological modifications at an earlier stage (Mollon and Da- 
nilova, 1996). This may also explain the fact that there is substantial further learning in the nonamblyopic eye. In the case of amblyopia, the cortical image is degraded and distorted in the amblyopic eye (Sireteanu et al., 2008). Although the stimulus configurations are the same, the visual brain may need to relearn a different, much sharper and more finely calibrated cortical image in the sound eye so as to extract the most salient visual features or signals for decision making that could, in part, explain the "secondary" learning of the same visual task.

Normal peripheral vision has been shown to be similar to amblyopic foveal vision in several respects and is, therefore, often used as a model to understand amblyopia (Levi et al., 1984, 1987; Wilson, 1991). In normal periphery, a wide range of visual functions are degraded relative to the fovea. We postulate that similar prolonged learning might also exist in normal periphery. Some evidence for prolonged learning of orientation discrimination in peripheral vision can be seen in the data of Furmanski and Engel (2000). This is particularly important for those who have reduced central vision and must rely on the remaining para-foveal vision.

In summary, our results show that the adult amblyopic brain retains a surprising degree of neural plasticity that is revealed by prolonged perceptual learning, and provide for the first time, documentation of the session-by-session dynamics of template retuning and noise reduction in amblyopic vision. We believe this prolonged learning does not occur in normal foveal vision in which visual learning is quickly completed in only a few hours, and that it has important potential clinical applications in visual rehabilitation of children and adults with amblyopia. In particular, our results point to the need for customizing the length or intensity of treatment based on the severity of amblyopia. We understand that there are individual variations in treatment efficacy and hence a large-scale multicenter clinical trial is necessary to quantify the dose-response relationship for different ages of onset, types and depths of amblyopia and optimize the treatment dose and schedule. Our present findings, together with other recent clinical studies, suggest that it may be the time to reconsider our notions about the critical period of neural plasticity in amblyopia.

\section{References}

Chung ST, Li RW, Levi DM (2006) Identification of contrast-defined letters in adults with amblyopia benefits from perceptual learning. Vision Res 46:3853-3861.

Chung STL, Li RW, Levi DM (2008) Learning to identify near-threshold luminance-defined and contrast-defined letters in observers with amblyopia. Vision Res 48:2739-2750.

Cleary M (2000) Efficacy of occlusion for strabismic amblyopia: can an optimal duration be identified? Br J Ophthalmol 84:572-578.

Dosher BA, Lu ZL (2007) The functional form of performance improvements in perceptual learning: learning rates and transfer. Psychol Sci 18:531-539.

Furmanski CS, Engel SA (2000) Perceptual learning in object recognition: object specificity and size invariance. Vision Res 40:473-484.

Gold J, Bennett PJ, Sekuler AB (1999) Signal but not noise changes with perceptual learning. Nature 402:176-178.

Green DM (1964) Consistency of auditory detection judgments. Psychol Rev 71:392-407.

Hess RF, Dakin SC, Tewfik M, Brown B (2001) Contour interaction in amblyopia: scale selection. Vision Res 41:2285-2296.

Huang CB, Zhou Y, Lu ZL (2008) Broad bandwidth of perceptual learning in the visual system of adults with anisometropic amblyopia. Proc Natl Acad Sci U S A 105:4068-4073.

Law CT, Gold JI (2008) Neural correlates of perceptual learning in a sensorymotor, but not a sensory, cortical area. Nat Neurosci 11:505-513.

Levi DM (2005) Perceptual learning in adults with amblyopia: a reevaluation of critical periods in human vision. Dev Psychobiol 46:222-232.

Levi DM (2008) Crowding-an essential bottleneck for object recognition: A mini-review. Vision Res 48:635-654.
Levi DM, Li RW (2008) Improving the performance of the amblyopic visual system. Philos Trans R Soc Lond B Biol Sci. Advance online publication. Retrieved December 9, 2008. doi:10.1098/rstb.2008.0203

Levi DM, Polat U (1996) Neural plasticity in adults with amblyopia. Proc Natl Acad Sci U S A 93:6830-6834.

Levi DM, Klein SA, Aitsebaomo P (1984) Detection and discrimination of the direction of motion in central and peripheral vision of normal and amblyopic observers. Vision Res 24:789-800.

Levi DM, Klein SA, Yap YL (1987) Positional uncertainty in peripheral and amblyopic vision. Vision Res 27:581-597.

Levi DM, Ciuffreda KJ, Selenow A (1991) Amblyopia: basic and clinical aspects. Boston: Butterworth-Heinemann.

Levi DM, Polat U, Hu YS (1997) Improvement in vernier acuity in adults with amblyopia. Practice makes better. Invest Ophthalmol Vis Sci 38:1493-1510.

Levi DM, Klein SA, Carney T (2000) Unmasking the mechanisms for Vernier acuity: evidence for a template model for Vernier acuity. Vision Res 40:951-972.

Li RW, Levi DM (2004) Characterizing the mechanisms of improvement for position discrimination in adult amblyopia. J Vis 4:476-487.

Li RW, Levi DM, Klein SA (2004) Perceptual learning improves efficiency by retuning the "template" for position discrimination. Nat Neurosci 7:178-183.

Li RW, Young KG, Hoenig P, Levi DM (2005) Perceptual learning improves visual perception in juvenile amblyopia. Invest Ophthalmol Vis Sci 46:3161-3168.

Li RW, Klein SA, Levi DM (2006) The receptive field and internal noise for position acuity change with feature separation. J Vis 6:311-321.

Li RW, Provost A, Levi DM (2007) Extended perceptual learning results in substantial recovery of both positional acuity and visual acuity in juvenile amblyopia. Invest Ophthalmol Vis Sci 48:5046-5051.

Mollon JD, Danilova MV (1996) Three remarks on perceptual learning. Spat Vis 10:51-58.

Nandy AS, Tjan BS (2007) The nature of letter crowding as revealed by firstand second-order classification images. J Vis 7:1-26.

Newman DK, East MM (2000) Prevalence of amblyopia among defaulters of preschool vision screening. Ophthalmic Epidemiol 7:67-71.

Polat U, Ma-Naim T, Belkin M, Sagi D (2004) Improving vision in adult amblyopia by perceptual learning. Proc Natl Acad Sci USA 101:6692-6697.

Polat U, Bonneh Y, Ma-Naim T, Belkin M, Sagi D (2005) Spatial interactions in amblyopia: effects of stimulus parameters and amblyopia type. Vision Res 45:1471-1479.

Sireteanu R, Bäumer CC, Iftime A (2008) Temporal instability in amblyopic vision: relationship to a displacement map of visual space. Invest Ophthalmol Vis Sci 49:3940-3954.

Smirnakis SM, Brewer AA, Schmid MC, Tolias AS, Schüz A, Augath M, Inhoffen W, Wandell BA, Logothetis NK (2005) Lack of long-term cortical reorganization after macaque retinal lesions. Nature 435:300-307.

Stewart CE, Moseley MJ, Stephens DA, Fielder AR (2004) Treatment doseresponse in amblyopia therapy: the Monitored Occlusion Treatment of Amblyopia Study (MOTAS). Invest Ophthalmol Vis Sci 45:3048-3054.

Stewart CE, Fielder AR, Stephens DA, Moseley MJ (2005) Treatment of unilateral amblyopia: factors influencing visual outcome. Invest Ophthalmol Vis Sci 46:3152-3160.

Westheimer G (2001) Is peripheral visual acuity susceptible to perceptual learning in the adult? Vision Res 41:47-52.

Wilson HR (1991) Model of peripheral and amblyopic hyperacuity. Vision Res 31:967-982.

Winship IR, Murphy TH (2008) In vivo calcium imaging reveals functional rewiring of single somatosensory neurons after stroke. J Neurosci 28:6592-6606.

Zhang B, Bi H, Sakai E, Maruko I, Zheng J, Smith EL 3rd, Chino YM (2005) Rapid plasticity of binocular connections in developing monkey visual cortex (V1). Proc Natl Acad Sci U S A 102:9026-9031.

Zhou XM, Merzenich MM (2007) Intensive training in adults refines A1 representations degraded in an early postnatal critical period. Proc Natl Acad Sci U S A 104:15935-15940.

Zhou Y, Huang C, Xu P, Tao L, Qiu Z, Li X, Lu ZL (2006) Perceptual learning improves contrast sensitivity and visual acuity in adults with anisometropic amblyopia. Vision Res 46:739-750. 